\title{
Postoperative infections: a preventable calamity
}

Qasim Mehmood ${ }^{1}$, Arisha Nawaz ${ }^{2}$, Priyanka Chahal ${ }^{3}$, Dattatreya Mukherjee ${ }^{*}$

1. King Edward Medical University, Lahore, Pakistan, qasimmehmood1051@gmail.com

2. Dow International University, Karachi, Pakistan, arisha.nawaz10@gmail.com

3. S. Tentishev Asian Medical Institute, Kyrgyzstan, drchahal10@gmail.com

4. Jinan University, Guangzhou, P.R China, dattatreyamukherjee4u@ outlook.com

*Corresponding Author: Dattatreya Mukherjee, Jinan University, P.R China dattatreyamukherjee4u@outlook.com

Type of Paper: Commentary

Reference Style: APA

Conflict of Interest: Authors don't have any COI

Funding: No Funding is attached 


\section{Introduction}

Surgical wounds generally heal by primary closure during which the wound edges are brought together, and is assisted by the use of sutures, stitches, staples, adhesive tape, or glue. Some of surgical wounds are more difficult to heal due to their anatomical position or an increased risk of infections ${ }^{[1]}$. When the surgical wound is not healed properly it leads to complications which include surgical site infections, dehiscence, and development of seromas or hematomas ${ }^{[2]}$. The infection appears in a wound created by a surgical or post-operative procedure of any cavity, bone, joint, tissue or prosthesis involved. The organisms involved in the infection are generally endogenous to the patient and come from the skin or any body part that has been opened. It is the most common post surgical complication, with increased morbidity and mortality ${ }^{[3]}$. In general surgery, reported rates of post surgical complications range from $6 \%$ to $44 \%{ }^{[4]}$. These infections are a common type of healthcare associated infections and frequent complication of hospitalization. They are responsible for prolonged hospital stay, increased admissions in intensive care units, readmission in hospitals after surgery, increased surgery costs, and delays to adjuvant systemic therapy ${ }^{[3,5]}$. The increased morbidity and costs associated with these postoperative complications motivate healthcare professionals to be vigilant in recognizing risk factors arising from patient comorbidities or circumstances of surgery ${ }^{[2]}$.

\section{Classification}

According to CDC definition, post surgical infections are classified by depth and tissue spaces involved. A superficial incisional surgical site infection involves only the skin or subcutaneous tissue, a deep incisional postoperative infection involves the fascia or muscular layers, and an organ space surgical site infection involves any part of the body opened or manipulated during a procedure, excluding the previously mentioned layers ${ }^{[6]}$.

\section{Diagnosis}

The diagnosis of postoperative infection poses a significant problem. Recent guidelines on post 
surgical infections indicate a lack of reliable methods to identify post-discharge SSI. The use of smartphone technology has revolutionized the clinical approach towards post surgical infections, whereby a patient can send daily photographs or updates of their wounds to the surgeon. Such self-reporting patient measures have been used as a tool for assessing patients after leaving the hospital. Wounds should be inspected regularly for fluid collection (haematoma or seroma), which may be an early feature of wound dehiscence. Wound dehiscence is defined as the separation of the layers of a surgical wound which may be superficial; partial; or complete, with separation of all layers and total disruption, often occurring after five and ten days of surgery ${ }^{[7]}$.

\section{Risk factors}

Various risk factors have been identified for the development of postoperative infection after surgery. These risk factors can be broadly separated into intrinsic or patient factors that are modifiable or nonmodifiable, as well as extrinsic factors e.g. procedure related, preoperative, and operative factors. Potentially modifiable intrinsic factors include blood sugar levels and diabetic status, dyspnoea, alcohol and smoking status, preoperative albumin levels, total bilirubin levels, obesity, and immunosuppression. Nonmodifiable intrinsic risk factors include increasing age, recent radiotherapy, and history of previous skin or soft tissue infection. Procedure-related risk factors include emergency and more complex surgery and wound classification. Facility risk factors include inadequate ventilation, increased operating room traffic, and inappropriate sterilization of the surgical equipment. Preoperative factors include pre-existing infection; inadequate skin preparation; hair removal; and antibiotic choice, administration, and duration. Intraoperative factors include duration of surgery, blood transfusion, maintenance of asepsis, hypothermia, poor-quality surgical hand scrubbing and gloving, and poor glycemic control of the patient $^{[6]}$

\section{Prevention:}

The prevention strategies are divided into three categories: preoperative, intra operative and postoperative. As a part of pre-operative measures, an electric razor with a single use tip should be used for hair removal ${ }^{[8]}$, obesity and malnutrition may present with a higher risk of postoperative infections and should be taken into account. According to Berthold et al, immunosuppression therapy impairs wound healing and increases infection risk ${ }^{[9]}$. The society for Healthcare epidemiology of America recommends perioperative cessation of immunosuppression therapy for as long as possible ${ }^{[10]}$. Antibiotic prophylaxis for clean/contaminated wounds and 
clean wounds with implanted foreign objects is required ${ }^{[11]}$. While a full course of antibiotics is indicated in contaminated, dirty wounds. Combined antibiotic prophylaxis (intravenous + oral) is more effective at preventing surgical site infections.

To combat intraoperative risk of infection, operating room is required to have zones of increasing sterility; or should be organized to have distinguished 'clean' and 'dirty' areas which should not cross, air conditioning should provide an exchange volume of 15-30 times the room volume and laminar air flow ${ }^{[12]}$. Skin decontamination with alcohol solutions of chlorhexidine gluconate and povidone iodine is required. Meanwhile the blood glucose level in both diabetic and non-diabetic patients should be $<200 \mathrm{mg} / \mathrm{dl}$ and body temperature should be maintained within normal limits. After operation, non-touch techniques, and use of sterile saline solution for rinse are used. Local antimicrobial products are not recommended.

\section{Management:}

The active management of post-operative surgical infections starts with taking wound swabs for culture and sensitivity, followed by empiric antibiotic therapy ${ }^{[13]}$. Microbiological studies are done when the patient is suffering from a severe clinical course, there is need for antibiotic therapy, there are suspected drug-resistant pathogens or there is allergy to first-line treatment. Empiric antibiotics are given when inflammation is beyond $5 \mathrm{~cm}$ with a fever of $>38.5^{\circ} \mathrm{C}$ and heart rate $>110 /$ min.

They can also be treated with antimicrobial products such as octenidine dihydrochloride. For staphylococcal infections first generation cephalosporins or fluoroquinolones can be used. For MRSA strains, linezolid and glycopeptides are more effective ${ }^{[14]}$. If the area is filled with pus, there is a need to drain the whole area in case of deep tissue infection while only partial drainage is required for superficial infections. Tissue debridement is indicated in case of necrosis. Negative pressure therapy can be considered for deep and non-healing wounds.

\section{Conclusion}

Mortality associated with post operative infections can be easily avoided with the right management. However, implementing prevention guidelines properly and taking pre operative and intra operative care can hinder the development of this complication in the first place.

\section{Acknowledgements:}

We want to thank our University, Professors and our parents for supporting us in this work. 


\section{Reference}

1. Webster, J., Liu, Z., Norman, G., Dumville, J. C., Chiverton, L., Scuffham, P., Stankiewicz, M., \& Chaboyer, W. P. (2019). Negative pressure wound therapy for surgical wounds healing by primary closure. The Cochrane database of systematic reviews, 3(3), CD009261. https://doi.org/10.1002/14651858.CD009261.pub4

2. Gabriel, A., Gupta, S., \& Orgill, D. P. (2019). Challenges and management of surgical site occurrences. Plastic and reconstructive surgery, 143(1S), 7S-10S.

3. da-Costa AC, Santa-Cruz F, Ferraz AAB. What's new in infection on surgical site and antibioticoprophylaxis in surgery? ABCD Arq Bras Cir Dig. 2020;33(4):e1558. DOI: /10.1590/0102-672020200004e1558

4. Rendell, V. R., Siy, A. B., Stafford, L. M. C., Schmocker, R. K., Leverson, G. E., \& Winslow, E. R. (2020). Severity of Postoperative Complications From the Perspective of the Patient. Journal of patient experience, 7(6), 1568-1576.

5. De Simone, B., Sartelli, M., Coccolini, F., Ball, C. G., Brambillasca, P., Chiarugi, M., ... \& Catena, F. (2020). Intraoperative surgical site infection control and prevention: a position paper and future addendum to WSES intra-abdominal infections guidelines. World journal of emergency surgery, 15(1), 10.

6. Ban, K. A., Minei, J. P., Laronga, C., Harbrecht, B. G., Jensen, E. H., Fry, D. E., ... \& Duane, T. M. (2017). American College of Surgeons and Surgical Infection Society: surgical site infection guidelines, 2016 update. Journal of the American College of Surgeons, 224(1), 59-74. 7. Sinha, S. (2019). Management of post-surgical wounds in general practice. Australian journal of general practice, 48(9), 596.

8. Alexander, J. W., Solomkin, J. S., \& Edwards, M. J. (2011). Updated recommendations for control of surgical site infections. Annals of surgery, 253(6), 1082-1093.

9. Berthold, E., Geborek, P., \& Gülfe, A. (2013). Continuation of TNF blockade in patients with inflammatory rheumatic disease. An observational study on surgical site infections in 1,596 elective orthopedic and hand surgery procedures. Acta orthopaedica, 84(5), 495-501.

10.nderson, D. J., Podgorny, K., Berríos-Torres, S. I., Bratzler, D. W., Dellinger, E. P., Greene, L., ... \& Kaye, K. S. (2014). Strategies to prevent surgical site infections in acute care hospitals: 
2014 update. Infection Control \& Hospital Epidemiology, 35(S2), S66-S88.

11. Kolasiński, W. (2018). Surgical site infections-review of current knowledge, methods of prevention. Pol Przegl Chir, 91(4), 41-47.

12. Spagnolo, A. M., Ottria, G., Amicizia, D., Perdelli, F., \& Cristina, M. L. (2013). Operating theatre quality and prevention of surgical site infections. Journal of preventive medicine and hygiene, 54(3), 131-137.

13. Yao, K., Bae, L., \& Yew, W. P. (2013). Post-operative wound management. Australian family physician, 42(12), 867-870.

14. Stevens, D. L., Bisno, A. L., Chambers, H. F., Dellinger, E. P., Goldstein, E. J., Gorbach, S. L., ... \& Wade, J. C. (2014). Practice guidelines for the diagnosis and management of skin and soft tissue infections: 2014 update by the Infectious Diseases Society of America. Clinical infectious diseases, 59(2), e10-e52. 